\title{
Nanotheranostics
}

2017; 1(2): 208-216. doi: 10.7150/ntno.18767

Research Paper

\section{Black Phosphorus Nanosheets for Mild Hyperthermia-Enhanced Chemotherapy and Chemo-Photothermal Combination Therapy}

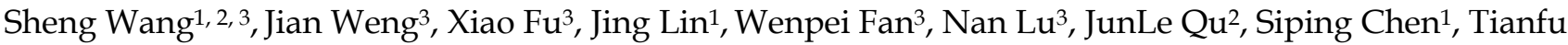 \\ Wang ${ }^{1}$, and Peng Huang ${ }^{1 凶}$ \\ 1. Guangdong Key Laboratory for Biomedical Measurements and Ultrasound Imaging, School of Biomedical Engineering, Health Science Center, Shenzhen \\ University, Shenzhen 518060, China: \\ 2. Key Laboratory of Optoelectronic Devices and Systems of Ministry of Education and Guangdong Province, College of Optoelectronic Engineering, \\ Shenzhen University, Shenzhen 518060, China; \\ 3. Laboratory of Molecular Imaging and Nanomedicine, National Institute of Biomedical Imaging and Bioengineering, National Institutes of Health, Bethesda, \\ Maryland 20892, United States. \\ $\triangle$ Corresponding author: peng.huang@szu.edu.cn
}

(c) Ivyspring International Publisher. This is an open access article distributed under the terms of the Creative Commons Attribution (CC BY-NC) license (https://creativecommons.org/licenses/by-nc/4.0/). See http://ivyspring.com/terms for full terms and conditions.

Received: 2016.12.14; Accepted: 2017.05.06; Published: 2017.05.30

\begin{abstract}
Near-infrared (NIR) light triggered photothermally enhanced cellular internalization strategy shows great potential to improve antitumor effect to fight against cancer. In this work, a black phosphorus (BP) nanosheet-based nanomedicine, which is composed of BP nanosheet, human serum albumin (HSA) and paclitaxel (PTX), was developed for mild hyperthermia-enhanced intracellular drug delivery and chemo-photothermal combination therapy. BP nanosheet was successfully synthesized by a one-pot biomimetic synthesis method using HSA as exfoliating agent and capping agent. Meanwhile, PTX can be efficiently loaded by HSA via hydrophobic interactions. The PTX-loaded HSA functionalized BP (BP-HSA-PTX) exhibits great photothermal performance, excellent biodegradability/biocompatibility, and effective drug loading. Upon NIR laser illumination, mild hyperthermia $\left(\sim 43^{\circ} \mathrm{C}\right)$ or photothermal effect can be achieved to improve the intracellular drug delivery efficiency and antitumor effect of BP-HSA-PTX. Our results indicate that HSA-functionalized BP has great potential for the loading, delivery, cellular uptake enhancement of chemotherapeutics and chemo-photothermal combination antitumor effect.
\end{abstract}

Key words: black phosphorus nanosheets, photothermal, mild hyperthermia, enhanced chemotherapy, combination therapy.

\section{Introduction}

Since many clinical chemotherapeutic drugs, such as paclitaxel (PTX) and doxorubicin (DOX), only act on intracellular targets [1-3], the enhanced intracellular drug delivery strategy plays a significant role in improving the chemotherapeutic effect. In recent years, various strategies have been explored to enhance the cellular uptake efficiency of drug-loaded nanoparticles [4-6]. For example, positive charge or ligand modification can enhance the cellular uptake of nanoparticles due to their strong binding to cell membranes by electrostatic or ligand-receptor interactions [7]. The hyperthermia method is another strategy to enhance intracellular delivery of chemotherapeutic drugs. When the tumor cells are heated to the designated temperature $\left(\sim 43^{\circ} \mathrm{C}\right)$, their cellular uptake efficiency would be significantly enhanced due to the increased cell membrane permeability $[8,9]$. This mild hyperthermia strategy shows good potential because the hyperthermia can be applied to tumor site locally without affecting normal tissues. Among the hyperthermia methods, near-infrared (NIR) light $(650-950 \mathrm{~nm})$ induced 
hyperthermia holds many merits of high safety, controllability and relatively low absorption of biological tissues and blood in this NIR region [10-14]. Furthermore, by adjusting the power density of NIR light, combined photothermal and chemotherapy also can be realized in this strategy. Photothermal conversion agents (PTCAs) are crucial moieties that absorb NIR light energy and convert it into heat, thus raising the local temperature [15-17]. However, the uses of many PTCAs are limited by their nonbiodegradability (e.g., noble metal nanomaterials, transition metal chalcogenides, carbon nanomaterials) or low photothermal stability (e.g., NIR dyes). Hence, the development of new PTCAs with excellent biodegradability/biocompatibility and photothermal stability is greatly demanded.

Black phosphorus (BP), one of the allotropes of phosphorus, is a new two dimensional layered material [18-20]. BP nanosheets with different thicknesses and sizes have been developed by mechanical and liquid exfoliation methods and applied in the electronic and optical fields [21-24]. However, little has been done to explore BP nanosheets for biomedical applications. Considering the excellent biodegradability/biocompatibility and the broad absorption across the ultraviolet (UV) and NIR region of BP nanomaterials [20], we expect that they may be great PTCAs to generate hyperthermia, which can be further applied in cancer therapy.

In this work, we report a PTX-loaded HSA functionalized BP (BP-HSA-PTX) for mild hyperthermia-enhanced chemotherapy and chemo-photothermal combination therapy. Firstly, BP nanosheet was prepared by one-pot biomimetic synthesis method using human serum albumin (HSA) as exfoliating/capping agents. In the exfoliation process, HSA macromolecules can bind to the surface of BP nanosheets. The HSA functionalized BP (BP-HSA) was purified by centrifugation. Secondly, PTX was loaded by BP-HSA via hydrophobic interactions between the hydrophobic domain on HSA and the PTX molecule. Finally, upon tuning NIR laser irradiation, BP nanosheets can convert the absorbed optical energy into heat, thus promoting the intracellular delivery of PTX. In our system, BP nanosheet was used as a photothermal agent to induce hyperthermia upon NIR laser irradiation. PTX was selected as a model anticancer drug. Most intriguingly, HSA plays triple roles such as exfoliating/capping agents of $\mathrm{BP}$ nanosheet and a carrier of PTX.

\section{Experimental section}

\section{Materials}

Black phosphorus (BP) powder was purchased from ACS Material (Medford, MA). Human serum albumin (HSA), methyl thiazolyl tetrazolium (MTT) and dimethyl sulfoxide (DMSO) was obtained from Sigma-Aldrich. Paclitaxel (PTX) was purchased from Biotang (Waltham, MA).

\section{One-pot biomimetic synthesis of BP-HSA-PTX}

The BP-HSA nanosheets were first prepared by one-pot biomimetic synthesis method using HSA-directed exfoliation of BP powder (Fig. 1A) [25]. In brief, $5 \mathrm{mg}$ of the BP powder was dispersed in 10 $\mathrm{mL}$ of pure water bubbled with nitrogen. Then HSA (50 mg) was added to the solution and the mixture was sonicated in ice bath for $12 \mathrm{~h}$. The resulting suspension was centrifuged for $5 \mathrm{~min}$ at $3000 \mathrm{rpm}$ to remove the unexfoliated large particles. The supernatant was further centrifuged for $10 \mathrm{~min}$ at $8000 \mathrm{rpm}$ and the resulting BP-HSA precipitate was collected and re-dispersed into water for further use and characterization. To prepare BP-HSA-PTX, $5 \mathrm{mg}$ of BP-HSA was dispersed in $2.5 \mathrm{~mL}$ of pure water. Then $20 \mu \mathrm{L}$ of PTX (20 mg/mL in DMSO) were added to the BP-HSA solution and stirred overnight in dark and then dialyzed against pure water (MWCO: 12-14 $\mathrm{kDa}$ ) for 2 days to remove DMSO and unbound PTX.

\section{Characterization of the nanosheets}

High Performance Liquid Chromatography (HPLC) (Agilent Technologies 1200) was used to determine the PTX loading efficiency of the BP-HSA-PTX. Tecnai TF30 transmission electron microscope (TEM) (FEI, Hillsboro, OR) and PicoForce Multimode atomic force microscopy (AFM) (Bruker, CA) were used to observe the size and morphology of the BP-HSA-PTX. Thermal gravimetric analysis (TGA) was employed to further determine the chemical composition of the BP-HSA. Genesys $10 \mathrm{~S}$ UV-Vis spectrophotometer (Thermo Scientific, Waltham, MA) were used to measure the UV-vis-NIR absorption spectra of the BP-HSA-PTX at room temperature. For the stability characterization, BP-HSA-PTX dispersed in water, PBS or DMEM + $10 \%$ FBS and incubated for $4 \mathrm{~h}$. To evaluate the biodegradability of BP, BP-HSA-PTX dispersed in water and incubated in air atmosphere. At appropriate time points, the absorbance of the solution was measured by UV-Vis spectrophotometer. 
A

A
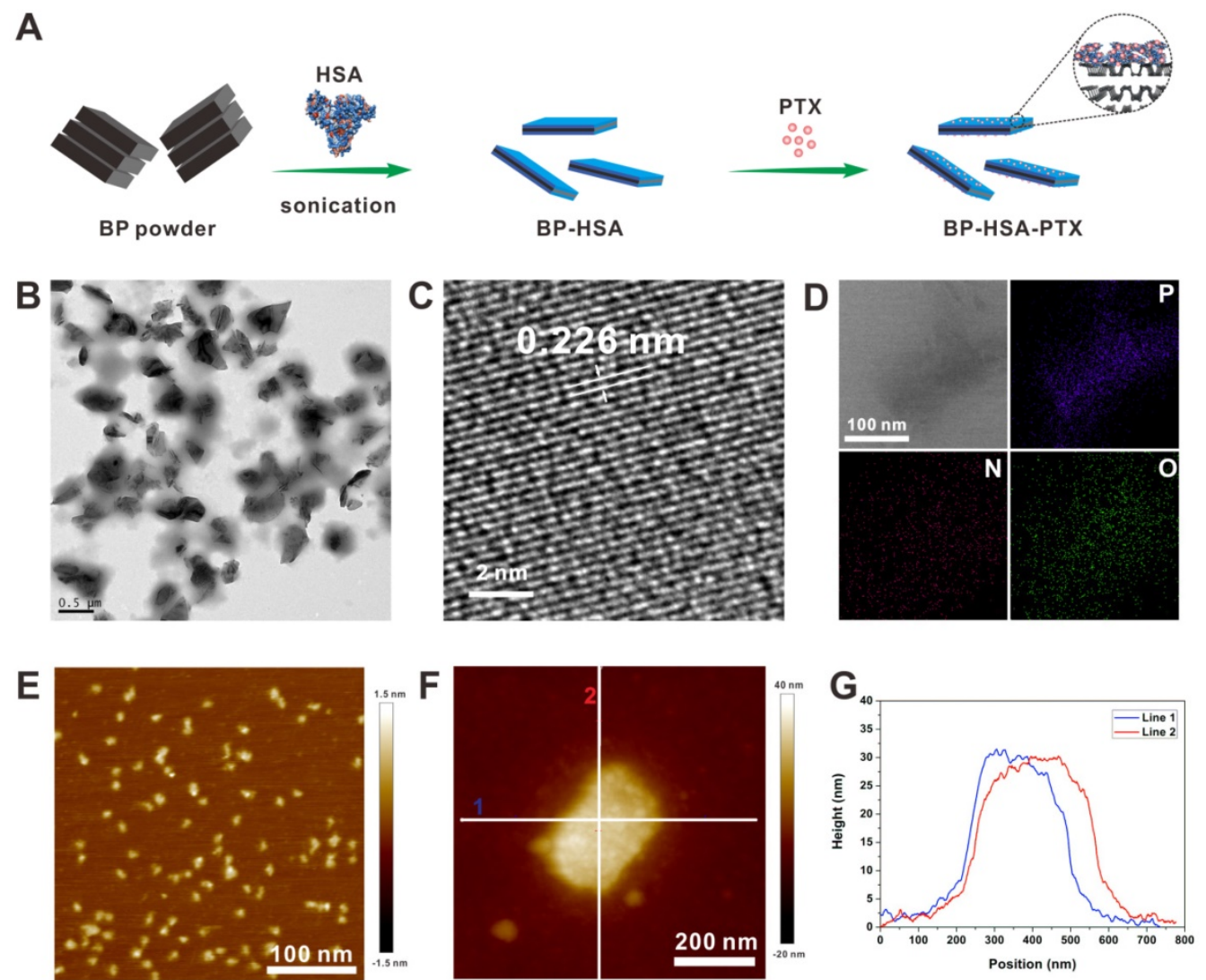

Figure 1. Preparation and characterization of the BP-HSA-PTX: (A) Preparation process; (B) TEM image; (C) HR-TEM image; (D) Element mapping; (E) AFM image of HSA; (F) AFM image of BP-HSA-PTX; (G) Height profiles along the white lines in Fig. 1F.

\section{Measurement of photothermal conversion performance}

To measure the photothermal conversion performance of the BP-HSA-PTX, $200 \mu \mathrm{L}$ of the BP-HSA-PTX aqueous solutions with different concentrations (i.e., $0.05,0.1$ and $0.2 \mathrm{mg} / \mathrm{mL}$ ) were placed in tubes and irradiated by an $808 \mathrm{~nm}$ NIR laser (power densities: $0.1 \sim 1.0 \mathrm{~W} / \mathrm{cm}^{2}$ ) for $10 \mathrm{~min}$. The real-time thermal images and temperature changes of the samples were recorded by a FLIR thermal camera. In this study, pure water was used as a control group. The photothermal conversion efficiency $(\eta)$ of the BP-HSA-PTX was determined by the following equations: $[12,26]$

$$
\begin{gathered}
\eta=\left(\mathrm{hS} \Delta \mathrm{T}_{\max }-\mathrm{Q}_{\mathrm{s}}\right) / \mathrm{I}\left(1-10^{-\mathrm{A} 808}\right) \\
\mathrm{hS}=\mathrm{mC} / \mathrm{\tau}_{\mathrm{s}}
\end{gathered}
$$

In the equations, $\mathrm{h}$ is the heat-transfer coefficient, $S$ is the surface area of the container, $\Delta T_{\max }$ is the temperature change of the solution at the maximum steady-state temperature, $Q_{s}$ is the heat associated with light absorption by the solvent. I is the laser power, A808 is the absorbance of the solution at 808 $\mathrm{nm}$. The variable $\tau_{s}$ is the sample-system time constant, and $\mathrm{m}$ and $\mathrm{C}$ are the mass and heat capacity of the deionized water used as the solvent.

\section{Mild hyperthermia-enhanced cellular internalization}

To observe the cellular uptake behavior of the BP-HSA-PTX with or without NIR light irradiation, fluorescein isothiocyanate (FITC) was used as the fluorescent substance. The preparation of FITC-labeled BP-HSA-PTX was the same as mentioned above except that HSA was replaced with FITC-conjugated HSA. U87MG cells were seeded in an 8 -well slide in $200 \mu \mathrm{L}$ of DMEM at $37^{\circ} \mathrm{C}$ under $5 \%$ $\mathrm{CO}_{2}$ atmosphere. Then FITC-labeled BP-HSA-PTX was added to the wells. After incubation for $30 \mathrm{~min}$ with (the power densities were adjusted to maintain the media temperature at $42.5 \pm 0.5^{\circ} \mathrm{C}$ ) or without NIR light irradiation, the cells were fixed and then stained with 4',6-diamidino-2-phenylindole (DAPI). Then the confocal images were acquired by an Olympus confocal microscope. To maintain the media temperature, pre-experiment need to be performed to determine the laser power scope. The media with a fixed concentration of BP were irradiated by NIR laser with different power densities and the temperature of the media was monitored by using an IR thermal imaging system. Thus an approximate power density 
that can raise the medium temperature to about $42{ }^{\circ} \mathrm{C}$ in $30 \mathrm{~min}$ was determined. In the cell experiments, we use the power density that determined above and the media temperature was also monitored by using an IR thermal imaging system. In the process of laser irradiation, the power density might be tuned slightly to ensure the temperature at $42.5 \pm 0.5^{\circ} \mathrm{C}$.

\section{In vitro cytotoxicity}

The cytotoxicity of the BP-HSA was determined by a MTT assay. U87MG human glioblastoma cells were seeded in a 96-well plate in $200 \mu \mathrm{L}$ of Dulbecco's modified Eagle's medium (DMEM) with 10\% fetal bovine serum (FBS) and incubated with BP-HSA with different concentrations (i.e., $0.1,0.2,0.3,0.4$ and 0.5 $\mathrm{mg} / \mathrm{mL}$ ) for $24 \mathrm{~h}$ under $5 \% \mathrm{CO}_{2}$ atmosphere. Then 20 $\mu \mathrm{L}$ of MTT solution $(5 \mathrm{mg} / \mathrm{mL})$ was added to each well. After another $4 \mathrm{~h}$ incubation at $37^{\circ} \mathrm{C}$, the culture media were removed and $200 \mu \mathrm{L}$ of DMSO was added to each well. The absorbance of each well was measured at a wavelength of $570 \mathrm{~nm}$. The group without BP-HSA incubation was used as a control. Then survival percentages were calculated by the following equation. Cell viability $(\%)=($ Absorbance value of treatment group/Absorbance value of control group) $\times 100 \%$.

\section{In vitro mild hyperthermia-enhanced chemotherapy}

To evaluate the in vitro mild hyperthermia-enhanced chemotherapy, U87MG cells were seeded in a 96-well plate in $100 \mu \mathrm{L}$ of DMEM with $10 \% \mathrm{FBS}$ and incubated at $37^{\circ} \mathrm{C}$ for $24 \mathrm{~h}$. Then the cells were incubated with different concentrations of BP-HSA-PTX with (the power densities were adjusted to maintain the media temperature at $42.5 \pm$ $0.5^{\circ} \mathrm{C}$ ) or without NIR light irradiation for $30 \mathrm{~min}$. Thereafter, the cells were washed with phosphate buffer solution (PBS) and incubated with fresh culture media for another $24 \mathrm{~h}$ at $37^{\circ} \mathrm{C}$. The relative cell viabilities were then measured by MTT assay as mentioned above. Meanwhile, the live/dead cells were costained with Calcein AM and propidium iodide (PI), and then imaged by an Olympus inverted microscope.

\section{In vitro combination therapy}

For in vitro chemo-photothermal combination therapy, U87MG cells were seeded in a 96-well plate in $100 \mu \mathrm{L}$ of DMEM with $10 \%$ FBS and incubated at 37 ${ }^{\circ} \mathrm{C}$ for $24 \mathrm{~h}$. Then the cells were incubated with PBS, BP-HSA or BP-HSA-PTX $(0.4 \mathrm{mg} / \mathrm{mL})$ for $2 \mathrm{~h}$, followed by exposure to an $808 \mathrm{~nm}$ laser at different power densities (i.e., $0.2,0.4,0.6,0.8,1.0$ and 1.2 $\mathrm{W} / \mathrm{cm}^{2}$ ) for $5 \mathrm{~min}$. Thereafter, the cells were washed with PBS and incubated with fresh culture media for another $24 \mathrm{~h}$ at $37^{\circ} \mathrm{C}$. The relative cell viabilities were then measured by MTT assay as mentioned above. The group without laser irradiation was used as a control.

\section{Results and Discussion}

\section{Preparation and characterization of the BP-HSA-PTX}

So far, BP nanomaterials were successfully prepared by several methods, such as mechanical or liquid exfoliation [20-22, 27]. However, those synthesis methods of BP nanomaterials usually require harsh environments or toxic organic solvents. Additionally, further surface modification of BP nanomaterials is necessary to achieve better water-solubility and stability for biomedical applications. Therefore, a facile, green, and biomimetic synthesis method of BP nanomaterials is highly desirable. In this study, we report a one-pot biomimetic method for effective exfoliation of BP in water by using HSA as an exfoliating agent and capping agent. The HSA not only can exfoliate the bulk BP into nanosheets, but also can serve as a capping agent to improve the stability of $\mathrm{BP}$ nanosheets. Meanwhile, the HSA can also serve as drug carriers, especially for loading/delivering PTX molecules.

The morphology of the BP-HSA-PTX was examined by TEM and AFM. As shown in Fig. 1B, the size of as-prepared BP-HSA-PTX was about $300 \mathrm{~nm}$. The high-resolution TEM (HRTEM) image showed clear lattice fringes of $0.226 \mathrm{~nm}$, which were attributed to the (014) plane of the BP crystal (Fig. 1C) [22]. The element mapping in Fig. 1D showed that the BP-HSA-PTX were composed of $\mathrm{P}, \mathrm{O}$ and $\mathrm{N}$ elements, indicating successful preparation of HSA modified BP nanosheets. The AFM analysis showed that the thickness of BP-HSA-PTX was about $30 \mathrm{~nm}$, which may be attributed to the HSA modification (Fig. 1E-G). The PTX loading efficiency in the BP-HSA-PTX was measured to be $4.2 \%$ by HPLC.

TGA was employed to further determine the chemical composition of the BP-HSA nanosheets. As shown in Fig. 2A, the bulk BP showed almost complete mass loss at around $500{ }^{\circ} \mathrm{C}$. In comparison to the bulk BP, the mass loss of BP-HSA in range of 200-400 ${ }^{\circ} \mathrm{C}$ represents the HSA decomposition. The stability of BP-HSA-PTX was also studied, as shown in Fig. 2B, the BP-HSA-PTX can be well dispersed in water and exhibit excellent stability in water, PBS and culture medium containing 10\% FBS, which may be attributed to the HSA surface modification. Furthermore, the BP nanomaterials can be degraded gradually in water [19]. As shown in Fig. 2C, during 
the 21 days observation period, the absorbance of the BP-HSA-PTX solution decreased with time, and the brown suspension became light in color, which indicated the degradation of the BP nanosheets. Indeed, the blood of animal is oxygen enriched environment and may lead to BP degradation. However, most of the blood oxygen was combined with hemoglobin, leading to a low free oxygen concentration. In this case, the degradation of $\mathrm{BP}$ in vivo is a relative long time process (approximately several weeks) and not affects its functions. Thus, BP-based nanomaterials show great potential for in vivo applications. The UV-vis-NIR absorption spectra showed that the BP-HSA-PTX had absorption in both UV and NIR regions with a good linear correlation between the absorbance at $808 \mathrm{~nm}$ and concentration (Fig. 2D), which similar to other 2D nanomaterials [28, 29].

\section{Photothermal conversion performance of the BP-HSA-PTX}

The photothermal conversion performance of the BP-HSA-PTX was investigated by measuring the temperature increase of BP-HSA-PTX suspension upon $808 \mathrm{~nm}$ NIR laser irradiation. Both BP-HSA-PTX concentration and laser power density dependent temperature increases were evaluated. As shown in Fig. 3A, when exposed to an $808 \mathrm{~nm}$ NIR laser $(1.0$ $\mathrm{W} / \mathrm{cm}^{2}$ ) for $10 \mathrm{~min}$, the temperature of BP-HSA-PTX
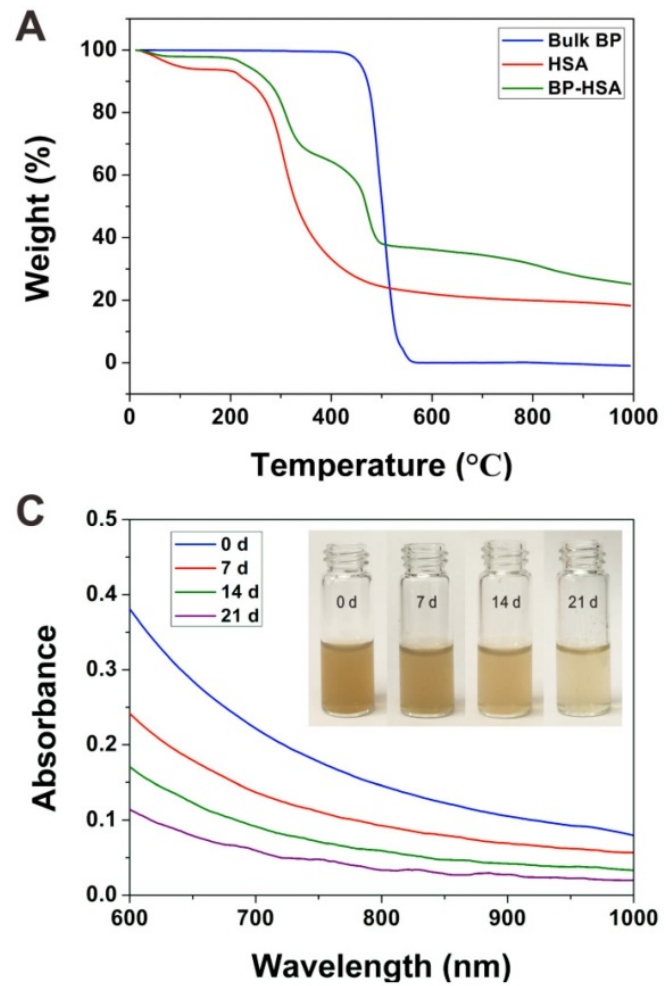

suspension increased rapidly. Even at a very low concentration $(0.2 \mathrm{mg} / \mathrm{mL})$, the temperature of BP-HSA-PTX solution can increased to more than 40 ${ }^{\circ} \mathrm{C}$ after $10 \mathrm{~min}$ of laser irradiation. In contrast, the pure water showed little temperature increase within $10 \mathrm{~min}$. These results indicated that the BP-HSA-PTX can convert NIR light into heat effectively. Meanwhile, the photothermal performances of BP-HSA-PTX $(0.1 \mathrm{mg} / \mathrm{mL})$ exposed to NIR laser at different power densities $\left(0.1-1.0 \mathrm{~W} / \mathrm{cm}^{2}\right)$ were also investigated. Fig. 3B showed that when the power density of exposed NIR laser was increased, the steady-state temperature of the BP-HSA-PTX suspension increased. To evaluate the photothermal stability of BP-HSA-PTX, the temperature changes of BP-HSA-PTX suspension with an NIR laser irradiation for $5 \mathrm{~min}$ (on) and without irradiation for additional $5 \mathrm{~min}$ (off) were monitored. As shown in Fig. 3C, in five laser on-off cycles, the photothermal effect of BP-HSA-PTX showed great stability without deterioration. Furthermore, after laser irradiation, the absorption spectra of BP-HSA-PTX showed little change, which further demonstrated the stability of BP-HSA-PTX (Fig. 3D). The photothermal conversion efficiency $(\eta)$ of the BP-HSA-PTX was determined to be $32.74 \%$ (Figure 3E and F), which is significantly higher than that of gold nanorods (21\%) [30].

B
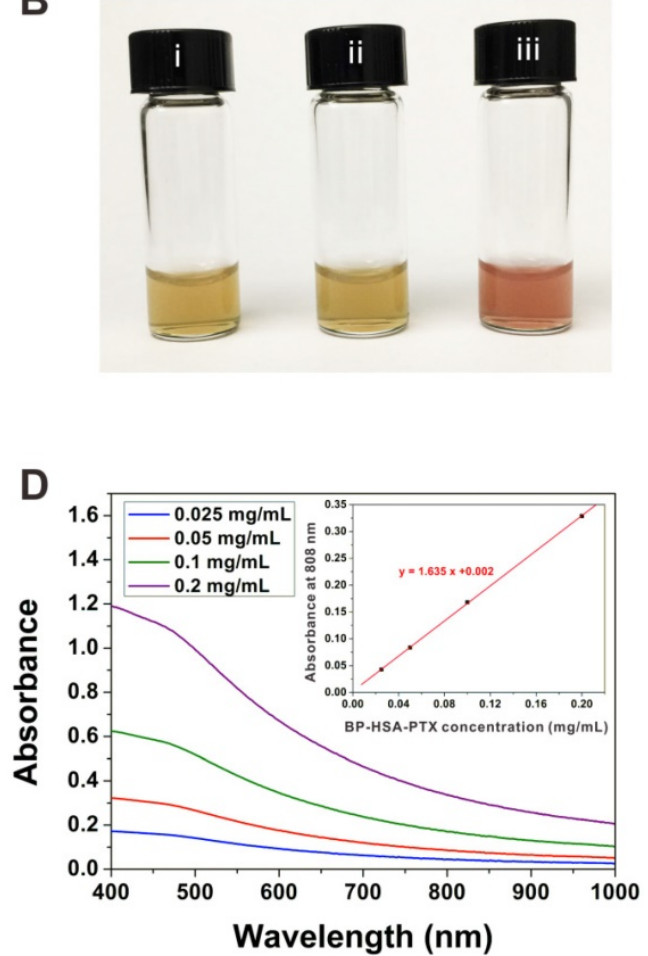

Figure 2. (A) TGA of BP, HSA and BP-HSA. (B) Photographs of BP-HSA-PTX dispersed in water (i), PBS (ii) and DMEM + 10\% FBS (iii). (C) Time-dependent changes in absorption spectra of BP-HSA-PTX solution. Inset: photographs of BP-HSA-PTX dispersed in water for different times. (D) Absorbance spectra of BP-HSA-PTX supernatant. Inset: The absorbance of BP-HSA-PTX at a wavelength of $808 \mathrm{~nm}$ as a function of the concentration. 

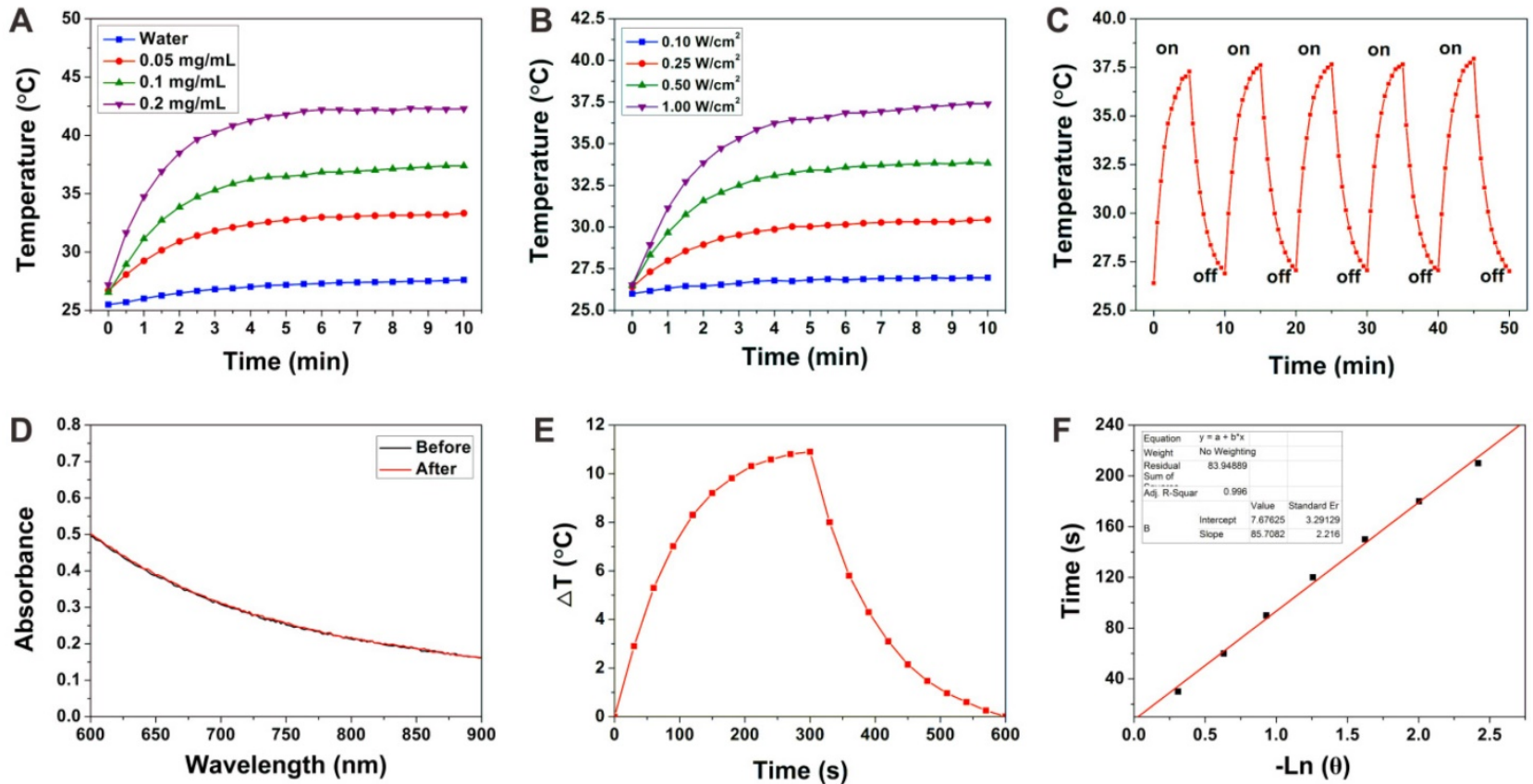

Figure 3. (A) Photothermal heating curves of pure water and the BP-HSA-PTX supernatants upon irradiation of an $808 \mathrm{~nm}$ laser $\left(1.0 \mathrm{~W} / \mathrm{cm}^{2}\right)$. (B) Photothermal heating curves of the BP-HSA-PTX $(0.1 \mathrm{mg} / \mathrm{mL})$ upon irradiation of an $808 \mathrm{~nm}$ laser at different power densities. (C) Heating of the BP-HSA-PTX supernatant for five laser on/off cycles. (D) Absorption spectra of BP-HSA-PTX before and after five laser on/off cycles. (E) Photothermal heating curve of the BP-HSA-PTX aqueous solution upon $808 \mathrm{~nm}$ laser irradiation $\left(1.0 \mathrm{~W} / \mathrm{cm}^{2}\right)$ and then the laser was shut off. F) Linear time data versus $-\ln \theta$ obtained from the cooling stage of Figure $3 \mathrm{E}$.
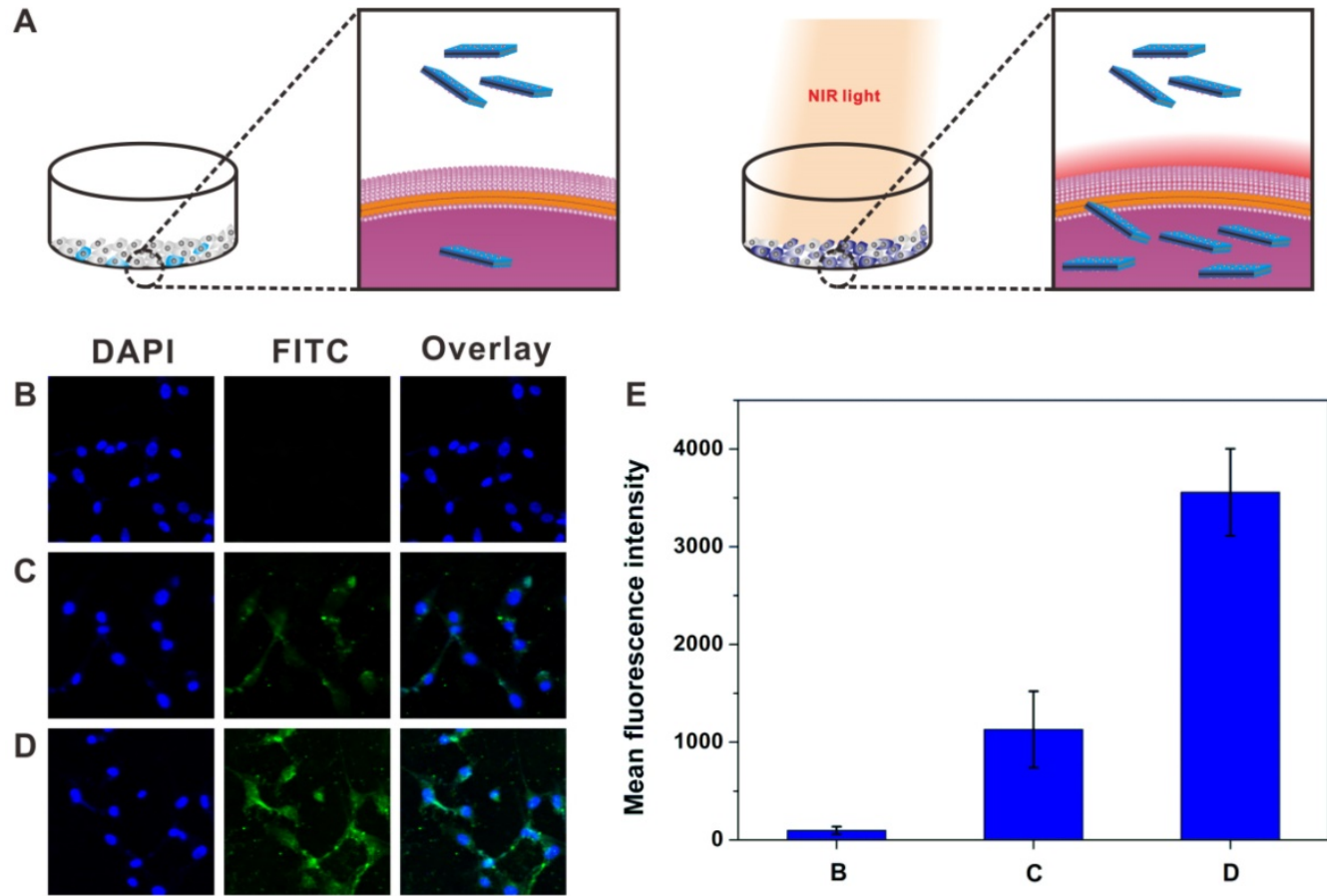

Figure 4. Schematic illustration (A) showing the photothermally enhanced cellular uptake of BP-HSA-PTX. Confocal images of U87MG cells incubated with PBS (B), FITC-labeled BP-HSA-PTX without (C) or with (D) NIR light irradiation (30 min). The temperature of cell medium was maintained at $42.5 \pm 0.5^{\circ} \mathrm{C}$ by adjusting the power density. (E) Mean FITC fluorescence intensity determined in cells in Figure 4B-D.

\section{Mild hyperthermia-enhanced cellular uptake}

It has been reported that enhanced cellular internalization of nanoparticles can be achieved by a mild photothermal heating $\left(\sim 43{ }^{\circ} \mathrm{C}\right)$ due to the increased permeability of cell membrane at this temperature [31, 32]. The cellular uptake behavior of FITC-labeled BP-HSA-PTX was assessed by using confocal microscope. As shown in Fig. 4A, U87MG cells were incubated with the FITC-labeled 
BP-HSA-PTX with or without NIR laser irradiation (30 min). To avoid hyperthermia-induced cell damage, the temperatures of cell media were maintained at $42.5 \pm 0.5{ }^{\circ} \mathrm{C}$ by adjusting the power densities of NIR laser. As shown in the confocal images (Fig. 4B-4D), significant increase of fluorescence intensity was observed in the cells incubated with the FITC-labeled BP-HSA-PTX under NIR light irradiation. Semiquantitative fluorescence intensity analysis of FITC in cells confirmed the higher uptake of FITC-labeled BP-HSA-PTX with laser irradiation than that without laser irradiation (Fig. 4E). These results indicated that the NIR light treatment can enhance the cellular uptake by photothermal effect.

\section{In vitro cytotoxicity}

For PTCAs and drug carrier materials, the biocompatibility is important property that influences their application and further clinical translation. U87MG cells were used to evaluate the cellular cytotoxicity of the BP-HSA. The concentration of samples ranged from 0.1 to $0.5 \mathrm{mg} / \mathrm{mL}$. As shown in Fig. 5, following $24 \mathrm{~h}$ incubation with BP-HSA solution, the viabilities of U87MG cells were still more than $80 \%$ even at a high concentration of $0.5 \mathrm{mg} / \mathrm{mL}$, suggesting good biocompatibility of the BP-HSA.

\section{In vitro mild hyperthermia-enhanced chemotherapy}

To assess the mild hyperthermia-enhanced intracellular delivery of PTX, U87MG cells were incubated with BP-HSA-PTX with or without NIR laser treatment (the temperatures of cell media were maintained at $42.5 \pm 0.5^{\circ} \mathrm{C}$ ). To serve as a control, U87MG cells were incubated with BP-HSA at the

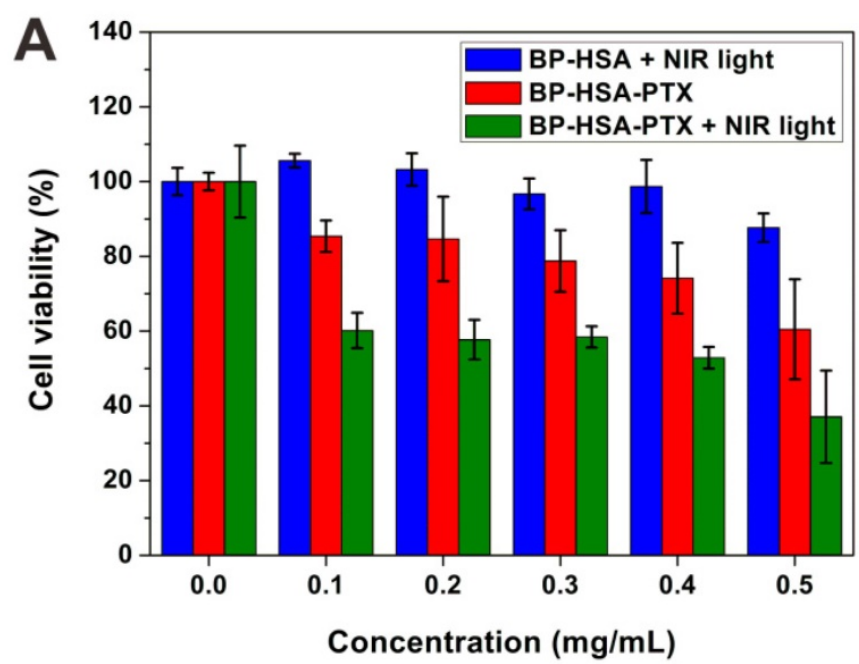

same concentration and irradiated with NIR laser. As shown in Fig. 6A, the BP-HSA with $30 \mathrm{~min}$ of NIR light irradiation showed low cytotoxicity even at a high concentration of $0.5 \mathrm{mg} / \mathrm{mL}$, which indicated the mild hyperthermia in such a short time didn't cause significant cell damage. In contrast, the cells incubated with the BP-HSA-PTX showed reduced cell viabilities due to the antitumor effect of PTX. It should be noted that the antitumor effects of BP-HSA-PTX with NIR light irradiation were higher than that of BP-HSA-PTX without NIR light irradiation. The calcein AM (green fluorescence, live cells) and PI (red fluorescence, dead cells) co-staining results (Fig. 6B) also confirmed the effective antitumor effect of the BP-HSA-PTX with NIR laser irradiation, which were consistent with the MTT results. These results may be attributed to the mild hyperthermia-enhanced intracellular drug delivery.

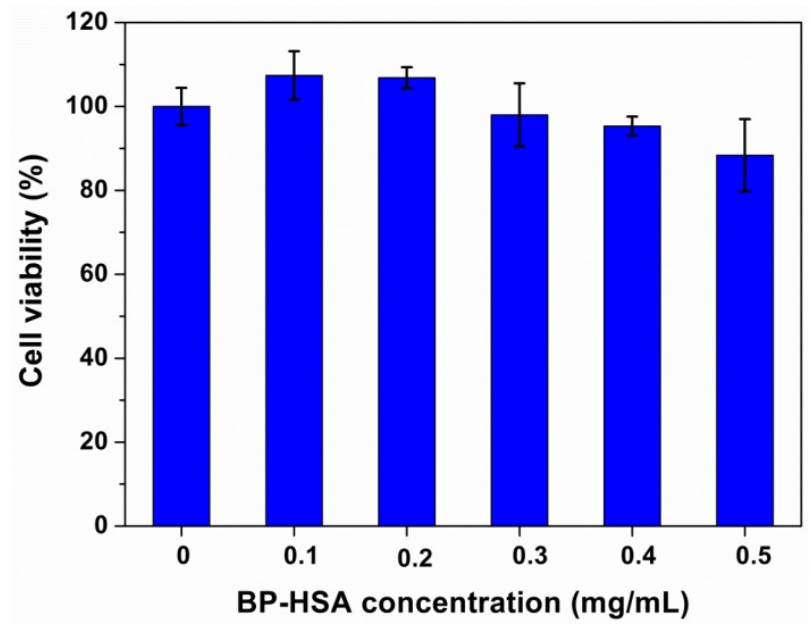

Figure 5. Viability of U87MG cells after incubation with BP-HSA for $24 \mathrm{~h}$.
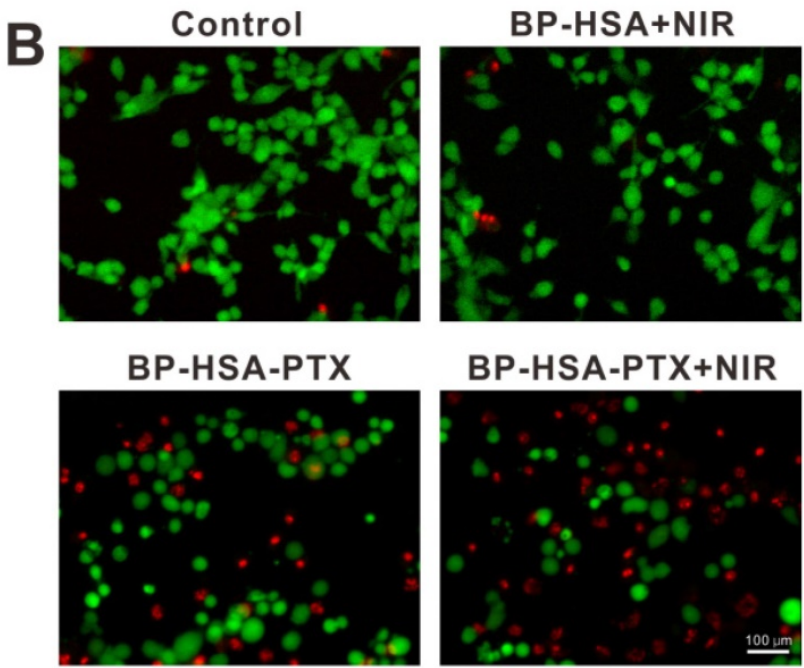

BP-HSA-PTX+NIR

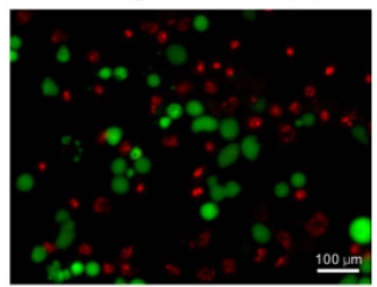

Figure 6. (A) Relative viabilities of U87MG cells after incubation with different samples with or without NIR light irradiation (30 min) and then washed with PBS and reincubated in fresh cell medium for $24 \mathrm{~h}$. (B) Fluorescence images of Calcein AM/PI costained U87MG cells after different treatments. 


\section{In vitro combination therapy}

Besides the mild hyperthermia-enhanced intracellular drug delivery, a chemo-photothermal combination therapy effect also can be achieved as we increased the power density of NIR laser [33]. To demonstrate the chemo-photothermal combination effect of BP-HSA-PTX at the cellular level, we tested the laser power density-dependent cancer cell killing at the same BP-HSA concentration. As shown in Fig. 7, for both BP-HSA and BP-HSA-PTX, increasing the laser power density would lead to enhanced photothermal cell killing. When the power density was increased to $0.8 \mathrm{~W} / \mathrm{cm}^{2}$, almost all the cells were effectively killed. As expected, compared with BP-HSA-PTX without laser irradiation and BP-HSA with laser irradiation, the BP-HSA-PTX with laser irradiation induced the most effective treatment efficacy, showing an obvious combination therapy effect.

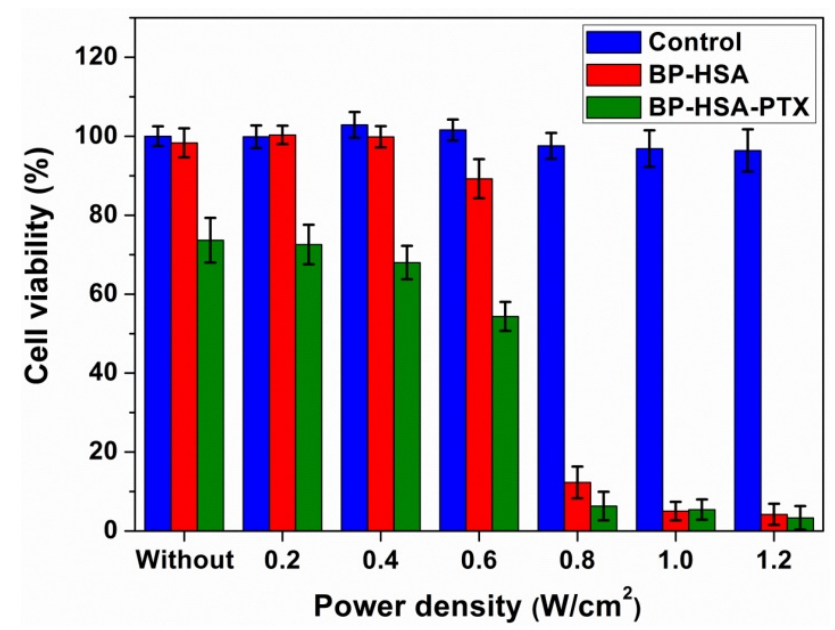

Figure 7. Relative viabilities of U87MG cells incubated with BP-HSA or BP-HSA-PTX $(0.4 \mathrm{mg} / \mathrm{mL})$ after laser irradiation at different power densities for $5 \mathrm{~min}$.

\section{Conclusions}

In this work, a novel black phosphorus nanosheet-based nanomedicine was developed for mild hyperthermia-enhanced chemotherapy and chemo-photothermal combination therapy. The BP-HSA nanosheets are prepared by one-pot biomimetic synthesis method using HSA as exfoliating/capping agents. Then PTX was loaded by BP-HSA via hydrophobic interactions between the hydrophobic domain on HSA and the PTX molecule. In our system, BP nanosheet is used as a PTCA to induce hyperthermia with excellent biodegradability and great photothermal stability. Most intriguingly, HSA plays triple roles such as exfoliating/capping agents of BP nanosheet and a carrier of PTX. Upon
NIR laser irradiation, the cellular uptake efficiency of BP-HSA-PTX can be markedly enhanced by the mild hyperthermia $\left(\sim 43^{\circ} \mathrm{C}\right)$, resulting in better antitumor effect. Furthermore, by adjusting the power density of NIR laser, chemo-photothermal combination therapy can be achieved to improve the antitumor effect of BP-HSA-PTX. Our results indicated that the BP-HSA has great potential for the loading, delivery, and cellular uptake enhancement of drug, gene, dye, or imaging contrast agents.

\section{Acknowledgment}

This work is financially supported by the startup fund from the Shenzhen University, the National Science Foundation of China $(81401465,51573096)$, the Postdoctoral Science Foundation of China (2016M600671), the Basic Research Program of Shenzhen (JCYJ20160422091238319) and the Intramural Research Program (IRP) of the NIBIB, NIH.

\section{Competing Interests}

The authors have declared that no competing interest exists.

\section{References}

1. Qiu L, Chen T, Ocsoy I, Yasun E, Wu C, Zhu G, et al. A cell-targeted, size-photocontrollable, nuclear-uptake nanodrug delivery system for drug-resistant cancer therapy. Nano Lett. 2015; 15: 457-63.

2. Chen Q, Liang C, Wang C, Liu Z. An imagable and photothermal "abraxane-like" nanodrug for combination cancer therapy to treat subcutaneous and metastatic breast tumors. Adv Mater. 2015; 27: 903-10.

3. Wang W, Cheng D, Gong F, Miao X, Shuai X. Design of multifunctional micelle for tumor-targeted intracellular drug release and fluorescent imaging. Adv Mater. 2012; 24: 115-20.

4. Cheng Z, Al Zaki A, Hui JZ, Muzykantov VR, Tsourkas A. Multifunctional nanoparticles: cost versus benefit of adding targeting and imaging capabilities. Science. 2012; 338: 903-10.

5. Wang $\mathrm{S}$, Huang $\mathrm{P}, \mathrm{Chen} X$. Hierarchical targeting strategy for enhanced tumor tissue accumulation/retention and cellular internalization. Adv Mater. 2016; 28: 7340-64.

6. Wang $H$, Zhao $P$, Liang $X$, Gong $X$, Song $T$, Niu R, et al. Folate-PEG coated cationic modified chitosan-cholesterol liposomes for tumor-targeted drug delivery. Biomaterials. 2010; 31: 4129-38.

7. Wang S, Huang P, Chen X. Stimuli-responsive programmed specific targeting in nanomedicine ACS Nano. 2016; 10: 2991-4.

8. Lu N, Huang P, Fan W, Wang Z, Liu Y, Wang S, et al. Tri-stimuli-responsive biodegradable theranostics for mild hyperthermia enhanced chemotherapy. Biomaterials. 2017; 126: 39-48.

9. Tian B, Wang C, Zhang S, Feng L, Liu Z. Photothermally enhanced photodynamic therapy delivered by nano-graphene oxide. ACS Nano. 2011; 5: 7000-9.

10. Cheng L, Wang C, Feng L, Yang K, Liu Z. Functional nanomaterials for phototherapies of cancer. Chem Rev. 2014; 114: 10869-939.

11. Shanmugam V, Selvakumar S, Yeh C-S. Near-infrared light-responsive nanomaterials in cancer therapeutics. Chem Soc Rev. 2014; 43: 6254-87.

12. Huang $\mathrm{P}$, Lin J, Li W, Rong P, Wang Z, Wang S, et al. Biodegradable gold nanovesicles with an ultrastrong plasmonic coupling effect for photoacoustic imaging and photothermal therapy. Angew Chem Int Ed. 2013; 52: 13958-64.

13. Huang $\mathrm{P}, \mathrm{Gao} Y$, Lin J, Hu H, Liao H-S, Yan X, et al. Tumor-specific formation of enzyme-instructed supramolecular self-assemblies as cancer theranostics. ACS Nano. 2015; 9: 9517-27.

14. Huang $\mathrm{P}$, Rong $\mathrm{P}$, Jin A, Yan X, Zhang MG, Lin J, et al. Dye-loaded ferritin nanocages for multimodal imaging and photothermal therapy. Adv Mater. 2014; 26: 6401-8.

15. Huang $P$, Rong $P$, Lin J, Li W, Yan X, Zhang MG, et al. Triphase interface synthesis of plasmonic gold bellflowers as near-infrared light mediated acoustic and thermal theranostics. J Am Chem Soc. 2014; 136: 8307-13.

16. Wang Z, Huang $\mathrm{P}$, Jacobson $\mathrm{O}$, Wang Z, Liu $\mathrm{Y}$, Lin $\mathrm{L}$, et al. Biomineralization-inspired synthesis of copper sulfide-ferritin nanocages as cancer theranostics. ACS Nano. 2016; 10: 3453-60. 
17. Lin J, Wang $\mathrm{M}, \mathrm{Hu} \mathrm{H}$, Yang $\mathrm{X}$, Wen $\mathrm{B}$, Wang $\mathrm{Z}$, et al. Multimodal-imaging-guided cancer phototherapy by versatile biomimetic theranostics with UV and $\gamma$-irradiation protection. Adv Mater. 2016; 28: 3273-9.

18. Zhang H. Ultrathin two-dimensional nanomaterials. ACS Nano. 2015; 9; 9451-69.

19. Wang H, Yang X, Shao W, Chen S, Xie J, Zhang X, et al. Ultrathin black phosphorus nanosheets for efficient singlet oxygen generation. J Am Chem Soc. 2015; 137: 11376-82.

20. Sun Z, Xie H, Tang S, Yu XF, Guo Z, Shao J, et al. Ultrasmall black phosphorus quantum dots: synthesis and use as photothermal agents. Angew Chem Int Ed 2015; 54: 11526-30.

21. Hanlon D, Backes C, Doherty E, Cucinotta CS, Berner NC, Boland C, et al. Liquid exfoliation of solvent-stabilized few-layer black phosphorus for applications beyond electronics. Nat Commun. 2015; 6: 8563 .

22. Guo Z, Zhang H, Lu S, Wang Z, Tang S, Shao J, et al. From black phosphorus to phosphorene: basic solvent exfoliation, evolution of raman scattering, and applications to ultrafast photonics. Adv Funct Mater. 2015; 25: 6996-7002.

23. Abbas AN, Liu B, Chen L, Ma Y, Cong S, Aroonyadet N, et al. Black phosphorus gas sensors. ACS Nano. 2015; 9: 5618-24.

24. Mayorga-Martinez CC, Sofer Z, Pumera M. Layered black phosphorus as a selective vapor sensor. Angew Chem Int Ed 2015; 127: 14525-8.

25. Guan G, Zhang S, Liu S, Cai Y, Low M, Teng CP, et al. Protein induces layer-by-layer exfoliation of transition metal dichalcogenides. J Am Chem Soc. 2015; 137: 6152-5.

26. Liu Y, Ai K, Liu J, Deng M, He Y, Lu L. Dopamine-melanin colloidal nanospheres: an efficient near-infrared photothermal therapeutic agent for in vivo cancer therapy. Adv Mater. 2013; 25: 1353-9.

27. Zhang X, Xie H, Liu Z, Tan C, Luo Z, Li H, et al. Black phosphorus quantum dots. Angew Chem Int Ed 2015; 54: 3653-7.

28. Cheng L, Liu J, Gu X, Gong H, Shi X, Liu T, et al. PEGylated WS $\mathrm{W}_{2}$ nanosheets as a multifunctional theranostic agent for in vivo dual-modal CT/photoacoustic imaging guided photothermal therapy. Adv Mater. 2014; 26: 1886-93.

29. Sheng Z, Song L, Zheng J, Hu D, He M, Zheng M, et al. Protein-assisted fabrication of nano-reduced graphene oxide for combined in vivo photoacoustic imaging and photothermal therapy. Biomaterials. 2013; 34: 5236-43.

30. Wang B, Wang JH, Liu Q, Huang $\mathrm{H}$, Chen $\mathrm{M}$, Li $\mathrm{K}$, et al. Rose-bengal-conjugated gold nanorods for in vivo photodynamic and photothermal oral cancer therapies. Biomaterials. 2014; 35: 1954-66.

31. Levi-Polyachenko NH, Merkel EJ, Jones BT, Carroll DL, Stewart JHt. Rapid photothermal intracellular drug delivery using multiwalled carbon nanotubes. Mol Pharm. 2009; 6: 1092-9.

32. Sherlock SP, Tabakman SM, Xie L, Dai H. Photothermally enhanced drug delivery by ultrasmall multifunctional $\mathrm{FeCo}$ /graphitic shell nanocrystals. ACS Nano. 2011; 5: 1505-12.

33. Tang Y, Hu H, Zhang MG, Song J, Nie L, Wang S, et al. An aptamer-targeting photoresponsive drug delivery system using "off-on" graphene oxide wrapped mesoporous silica nanoparticles. Nanoscale. 2015 ; 7: 6304-10. 